\title{
Radioguided surgery with 68Ga-DOTATATE for patients with neuroendocrine tumors
}

\author{
Valentina Ambrosini, Stefano Fanti \\ Nuclear Medicine, DIMES, University of Bologna, S.Orsola-Malpighi Hospital, Bologna, Italy \\ Correspondence to: Stefano Fanti, MD. Nuclear Medicine, DIMES, University of Bologna, S.Orsola-Malpighi Hospital, 40138 Bologna, Italy. \\ Email: stefano.fanti@aosp.bo.it. \\ Comment on: El Lakis M, Gianakou A, Nockel P, et al. Radioguided Surgery With Gallium 68 Dotatate for Patients With Neuroendocrine Tumors. \\ JAMA Surg 2019;154:40-5.
}

Submitted Jun 03, 2019. Accepted for publication Jun 11, 2019.

doi: 10.21037/hbsn.2019.06.04

View this article at: http://dx.doi.org/10.21037/hbsn.2019.06.04

Targeted precision neuroendocrine neoplasms (NEN) surgery may represent an innovative, yet promising, development of surgical procedures to achieve a radical resection of the primary tumour (by intra-operative confirmation of tumour-free margins) and to improve the identification of small sized lesions (that might otherwise be missed by either pre-operative functional imaging, ultrasound or manual palpation during surgery). From a practical point of view, radioguided surgery (RGS) requires a close collaboration between the nuclear medicine and the surgical teams, so that a small amount of radiopharmaceutical is intravenously injected in the patient right before surgery. The tumour lesions' emitted radiation is then detected by a hand-held probe by the surgeon intra-operatively.

RGS is routinely and successfully used in breast (1) and melanoma (2) patients as well as for localization of hyperfunctioning parathyroid glands (3).

Previous reports of the employment of RGS in NEN first employed 99Tcm-labelled somatostatin analogues (SA) $(4,5)$. However, the development of beta-emitting SA for PET/CT imaging (SA-PET/CT) of well differentiated NEN tumours rendered pure scintigraphy imaging obsolete. The higher accuracy of SA-PET/CT was reported in many papers $(6,7)$ and their theranostic potential (by labelling SA compounds with beta-minus isotopes for target treatment) also contributed to their increasing use (8).

In 2015, a prospective study on a relatively small cohort $(n=14)$ of gastroentero-pancreatic (GEP)-NEN patients with positive pre-operative $68 \mathrm{Ga}$-DOTATATE $\mathrm{PET} /$ $\mathrm{CT}$ was performed to assess the added value of $68 \mathrm{Ga}-$
DOTATATE RGS (5 mCi of radiopharmaceutical were administered at start of surgery in the operating room) (9). The authors reported that $68 \mathrm{Ga}$-DOTATATE RGS had the highest correct identification by pathology for gastric and small bowel NEN, including mesenteric lymph nodes, correctly identifying more than $80 \%$ of lesions. The reported concordance between surgeon's palpation and RGS was almost perfect, with only $3 / 35$ (8.6\%) lesions detected by RGS-only (9). It is interesting to notice, however, that the 3 above mentioned lesions were all at ileal level and millimetric in size, suggesting that the setting in which RGS might show a higher added value is definitely the identification of small mid-gut lesions. In fact, surgeons usually use manual palpation or intra-operative ultrasound to identify small ileal NEN lesions but their correct detection can be challenged, even in case of experienced surgeons, by their often millimetric size and multiple localizations in the same patient.

The intraoperative assessment of MEN patients, that are expected to develop multiple NEN lesions, is another potentially interesting setting in which RGS might prove additional value. Moreover, already surgically treated patients might be candidates for RGS to improve the identification of still viable tumour cells within surgical scar tissue, multiple adhesions or altered-anatomy regions.

In a previous report by Kaemmerer et al. (10) (9 NEN patients with mostly mid-gut primary tumour, stage 4), RGS detected $94 \%$ of the whole lesions as compared to pre-surgical PET/CT (69\%) and surgical palpation (50\%).

Considering the high background activity of the spleen, the adrenals and, to a lesser extent, the liver, RGD seems 
not appropriate to identify small pancreatic tail NEN or liver secondary lesions $(9,10)$.

The paper by El Lakis et al. recently published on FAMA Surgery 2019 (11), described a prospective cohort of 44 NEN patients $(59 \%$ with a genetic predisposition to develop NEN) who underwent 68Ga-DOTATATE RGS $(5 \mathrm{mCi}$ were administered intravenously at the time of the operation). The authors reported a high RGS accuracy for true NEN lesions detection (with the more frequent disease sites being the nodes, followed by pancreas, small bowel, liver and adrenal/paraganglioma). The tumour-tobackground (TBR) threshold of 2.5 yielded the highest sensitivity (90\%). They also found that the best intraabdominal organ for TBR optimization was the omentum (easily accessible, showing a homogeneous/minimal uptake and rarely involved with NEN carcinomatosis). Overall, 5/39 (13\%) lesions were detected by RGS-only as compared to pre-surgical PET/CT, intraoperative ultrasound and palpation. Last but not least, the radiation exposure to the operating room personnel was reported to be negligible. The authors concluded that RGS is safe and can provide additional value in NEN lesions' detection when the TBR (using the omentum as background) is above 2.5.

Overall, the results portrayed on the accuracy and safety of $68 \mathrm{Ga}$-DOTATATE RGS in NEN are very promising, however, several issues still need to be investigated. First of all, since the estimated number of additional lesions detected by RGS-only seems to be approximately $10 \%$ across studies, the identification of the patients' subset who might benefit more from the procedures seems mandatory. From the published, preliminary studies it seems that mid-gut NET patients with an indication to elective surgery, patients with known NEN secondary lesions and suspected/unidentified ileal primary or patients with genetic predisposition to develop NEN might be the ideal candidates. Secondly, a more in-depth analysis should clarify whether the performance of RGS increases the number of radical resections, to what extent the performance of RGS impacts the surgical procedure itself and survival. Finally, considering the high prevalence of pancreatic NEN primary and small liver NEN lesions, a deeper evaluation of the possibility to identify lesions close to/within high background parenchymas (e.g., spleen, adrenal glands and kidneys) should be further investigated.

\section{Acknowledgments}

None.

\section{Footnote}

Conflicts of Interest: The authors have no conflicts of interest to declare.

Ethical Statement: The authors are accountable for all aspects of the work in ensuring that questions related to the accuracy or integrity of any part of the work are appropriately investigated and resolved.

\section{References}

1. Giammarile F, Alazraki N, Aarsvold JN, et al. The EANM and SNMMI practice guideline for lymphoscintigraphy and sentinel node localization in breast cancer. Eur J Nucl Med Mol Imaging 2013;40:1932-47.

2. Bluemel C, Herrmann K, Giammarile F, et al. EANM practice guidelines for lymphoscintigraphy and sentinel lymph node biopsy in melanoma. Eur J Nucl Med Mol Imaging 2015;42:1750-66.

3. Hindié E, Ugur O, Fuster D, et al. 2009 EANM parathyroid guidelines. Eur J Nucl Med Mol Imaging 2009;36:1201-16.

4. Modlin IM, Cornelius E, Lawton GP. Use of an isotopic somatostatin receptor probe to image gut endocrine tumors. Arch Surg 1995;130:367-73; discussion 373-4.

5. Hall NC, Nichols SD, Povoski SP, et al. Intraoperative Use of a Portable Large Field of View Gamma Camera and Handheld Gamma Detection Probe for Radioguided Localization and Prediction of Complete Surgical Resection of Gastrinoma: Proof of Concept. J Am Coll Surg 2015;221:300-8.

6. Etchebehere EC, de Oliveira Santos A, Gumz B, et al. 68Ga-DOTATATE PET/CT, 99mTc-HYNIC-octreotide SPECT/CT, and whole-body MR imaging in detection of neuroendocrine tumors: a prospective trial. J Nucl Med 2014;55:1598-604.

7. Geijer H, Breimer LH. Somatostatin receptor PET/ $\mathrm{CT}$ in neuroendocrine tumours: update on systematic review and meta-analysis. Eur J Nucl Med Mol Imaging 2013;40:1770-80.

8. Bozkurt MF, Virgolini I, Balogova S, et al. Guideline for $\mathrm{PET} / \mathrm{CT}$ imaging of neuroendocrine neoplasms with 68Ga-DOTA-conjugated somatostatin receptor targeting peptides and 18F-DOPA. Eur J Nucl Med Mol Imaging 2017;44:1588-601.

9. Sadowski SM, Millo C, Neychev V, et al. Feasibility of Radio-Guided Surgery with ${ }^{68}$ Gallium-DOTATATE in 
Patients with Gastro-Entero-Pancreatic Neuroendocrine Tumors. Ann Surg Oncol 2015;22 Suppl 3:S676-82.

10. Kaemmerer D, Prasad V, Daffner W, et al. Radioguided surgery in neuroendocrine tumors using Ga-68-labeled somatostatin analogs: a pilot study. Clin Nucl Med

Cite this article as: Ambrosini V, Fanti S. Radioguided surgery with $68 \mathrm{Ga}$-DOTATATE for patients with neuroendocrine tumors. Hepatobiliary Surg Nutr 2020;9(1):67-69. doi: 10.21037/ hbsn.2019.06.04
2012;37:142-7.

11. El Lakis M, Gianakou A, Nockel P, et al. Radioguided Surgery With Gallium 68 Dotatate for Patients With Neuroendocrine Tumors. JAMA Surg 2019;154:40-5. 